\title{
La sistematización de experiencias, una investigación social cualitativa que potencia buenas prácticas de convivencia y gobierno. La experiencia de un conjunto residencial multifamiliar en Cali, Colombia
}

\author{
Martha Lucia Echeverry-Velásquez \\ Magíster en Sociología. Trabajadora Social \\ Universidad del Valle. Cali, Colombia \\ https://orcid.org/0000-0001-7381-9787• martha.echeverry@correounivalle.edu.co \\ Manuela Prada-Dávila \\ Trabajadora Social \\ Universidad del Valle. Cali, Colombia \\ https:/ / orcid.org/0000-0002-6049-5178 • manuela.prada@correounivalle.edu.co
}

Resumen

En este artículo se aborda el tema de la sistematización de experiencias como opción investigativa generadora de trasformaciones, por cuanto promueve procesos de reflexividad que potencian a su vez, cambios positivos en actitudes, prácticas y relaciones. Para esto se presenta la sistematización de una experiencia en convivencia y gobierno privado en un multifamiliar de la ciudad de Cali. Los fundamentos teóricos y metodológicos, presentados en el artículo, se basan en los postulados de la investigación social cualitativa y en los aportes de diferentes autores que han trabajado la relación entre sistematización e investigación, a partir de las experiencias que impactan significativamente la vida de los sujetos.

Es así como a través de la reconstrucción, interpretación crítica y potenciación de la experiencia en el multifamiliar, sus habitantes hicieron parte de un proceso de reflexiones individuales y colectivas sobre el ejercicio de gobierno, desde el cual se regula la vida y la convivencia en su espacio de residencia, permitiendo así, reconocer y apropiar aprendizajes sobre la participación, organización comunitaria, la construcción de democracia desde espacios micro sociales y las relaciones basadas en el pluralismo. Todo esto representa un aporte a la comprensión sobre el fenómeno del encerramiento residencial en Colombia, cuya interpretación, desde los estudios de vida cotidiana, contribuyen al desarrollo humano y a procesos de construcción de convivencia civilista y gobierno democrático.

Palabras clave: Sistematización de experiencias; Investigación cualitativa; Convivencia; Gobierno privado; Conjuntos residenciales multifamiliares.

Recibido: 04/09/2020 | Aprobado: 13/11/2020 | Publicado: 01/01/2021

(i) (-) Esta obra está bajo una Licencia Creative Commons Atribución-NoComercial-CompartirIgual 4.0 Internacional.

Financiación o proveniencia del artículo: Este artículo se deriva del proyecto de investigación Convivencia y Gobernabilidad en Multifamiliares, financiado por la Universidad del Valle, Cali, Colombia.

¿Cómo citar este artículo? / How to quote this article?

Echeverry-Velásquez, M. L., y Prada-Dávila, M. (2021). La sistematización de experiencias, una investigación social cualitativa que potencia buenas prácticas de convivencia y gobierno. La experiencia de un conjunto residencial multifamiliar en Cali, Colombia. Prospectiva. Revista de Trabajo Social e intervención social, (31), $151-176$. doi: $10.25100 /$ prts.v0i31.10595. 
Echeverry-Velásquez y Prada-Dávila

\title{
The systematization of experiences, a qualitative social investigation that promotes good practices of coexistence and governance. The experience of a gated community in Cali, Colombia
}

\begin{abstract}
This article responds to the need to address the systematization of experiences as an investigative option that generates transformations, because it promotes processes of reflexivity that enhance positive changes in attitudes, practices and relationships. In this sense, the exercise of systematization of an experience of coexistence and private government in a residential estate in the city of Cali is presented. The theoretical and methodological foundations are based on the postulates of qualitative social investigation and on the contributions of different authors who have worked on the relationship between systematization and investigation, based on experiences that significantly impact the lives of the subjects. Thus, through the reconstruction, critical interpretation and enhancement of their experience of life in a residential estate, its inhabitants were part of a process of individual and collective reflections on the exercise of government from which life and coexistence in their community are regulated. The experience allowed us to recognize and learn about participation, community organization, the construction of democracy from micro-social spaces and relationships based on pluralism. All this represents a contribution to the understanding of the phenomenon of residential estates in Colombia, whose interpretation, from the studies of daily life, promote human development and the processes of building healthy coexistence and democratic government.

Keywords: Systematization of experiences; Qualitative research; Coexistence; Private government; Gated communities.

Sumario: 1. Introducción, 2. Antecedentes y fundamentos de la sistematización de experiencias como opción investigativa, 3. Acercamiento a los modelos de sistematización de experiencias, al modelo y propuesta metodológica de la experiencia objeto de estudio, 4 . Metodología, 4.1 Reconstrucción, interpretación y potenciación de una experiencia en convivencia y gobierno en un multifamiliar, 4.2. Reconstrucción de la experiencia en convivencia y gobierno, 5. Hallazgos, 5.1 Interpretación de la experiencia en convivencia y gobierno, 5.2. Potenciación de la experiencia en convivencia y gobierno, 6. Conclusiones, 7. Referencias bibliográficas.
\end{abstract}




\section{Introducción}

Con la intención de aportar a las reflexiones sobre la Sistematización de experiencias como opción investigativa, en este artículo se hará un acercamiento a la implementación de la sistematización de experiencias en el marco del estudio cualitativo Convivencia y Gobernabilidad en Multifamiliares, para esto se mostrará el proceso que se llevó a cabo para sistematizar una experiencia en convivencia y gobierno privado en un multifamiliar de la ciudad de Cali1, en el periodo 2011-2012, desde el modelo del PESEP2. De esta forma, el núcleo del texto radica en la aproximación reflexiva al ejercicio de reconstrucción, interpretación y potenciación de la experiencia sistematizada, por lo cual no se muestran en profundidad los resultados del estudio, sino, que se presentan de forma sintética y en su relación con la forma en que se llegó a estos, desde las diferentes etapas y actividades que se promueven desde la sistematización de experiencias como investigación cualitativa.

Para comprender donde se sitúa la sistematización de la experiencia en gobierno privado, es importante hacer alusión al escenario de los multifamiliares. Estos son también llamados conjuntos o unidades residenciales, además de otros términos con los que se les reconoce 3 , son bienes inmobiliarios para vivienda en la modalidad de encerramiento residencial. Este tipo de emprendimiento habitacional es el resultado de un fenómeno de carácter mundial, donde la segregación espacial y los barrios encerrados son un producto del mercado inmobiliario que se relacionan con una respuesta ante la inseguridad, por esto su acceso es restringido a través de dispositivos de vigilancia ${ }^{4}$ (Rincón-Salazar, MaldonadoGómez y Echeverry-Velásquez, 2009). En ellos también confluyen los bienes de propiedad exclusiva, como son los apartamentos que integran los conjuntos residenciales y los bienes de copropiedad, tales como las zonas comunes y los mobiliarios que en estas se encuentran.

Los copropietarios al interior de los multifamiliares además de compartir la propiedad sobre los bienes comunes, comparten la responsabilidad sobre la gestión y administración de estos, así como sobre la convivencia entre los residentes; esto de acuerdo con EcheverryVelásquez y Prada-Dávila (2017) implica sistemas de gobierno para la regulación de la vida que se comparte al interior de los multifamiliares. En Colombia existe la Ley 675 de 2001,

1 Multifamiliar ubicado al sur de la ciudad de Cali, grupo socioeconómico medio-alto, conformado por 219 apartamentos y construido en un periodo de tiempo que data de 1993 a 2008.

2 Programa de Investigación sobre Experiencias Significativas de Educación Popular de Adultos (PESEP) del Grupo Interuniversitario de Trabajo en Educación Popular (GIU) conformado por grupos de profesores de las Facultades de Educación de las Universidades de Antioquía, Cauca, Pedagógica Nacional, Surcolombiana y del Valle (Carvajal-Burbano, 2006, p. 73).

3 Barrios cerrados, enclaves, condominios, entre otros.

4 Por esta modalidad de vivienda no sólo optan las clases socioeconómicas alta y media -alta por razones de seguridad- y segregación frente a otras poblaciones, pues se ha vuelto también el tipo de vivienda mayormente ofertado por los programas de vivienda subsidiados por el Estado, convirtiéndose igualmente en opción para los ciudadanos con ingresos medios y medio bajos. 
que contempla el régimen de propiedad horizontal y se constituye en el marco normativo que estipula el gobierno privado en los multifamiliares, en tanto regula esta forma de propiedad "con el fin de garantizar la seguridad y la convivencia pacífica en los inmuebles sometidos a ella, así como la función social de la propiedad" (p. 1). Conforme al régimen de propiedad horizontal $(\mathrm{PH})$, la persona jurídica $(\mathrm{PJ})$ del multifamiliar la conforman los propietarios de bienes de dominio particular al interior del multifamiliar y en este debe existir un reglamento de propiedad horizontal en particular, dicho reglamento debe regular la administración, dirección y control del conjunto residencial.

A continuación, se ilustran las instancias de administración y gobierno en los multifamiliares.

\begin{abstract}
Según el art 32 de la citada ley, el objeto de la persona jurídica apunta al ejercicio del gobierno de la $\mathrm{PH}$, en cabeza de quienes dirigen y administran. El art 36, establece que la dirección y administración de la PJ corresponde a: 1) Asamblea General de Copropietarios, órgano de dirección (art. 38) y máxima expresión, toma las decisiones que atañen al conjunto residencial. 2) Consejo de Administración, que ejerce la dirección del conjunto, en ausencia o representación de la Asamblea General de Copropietarios, toma las determinaciones para que la persona jurídica cumpla sus fines (art. 55) y debe actuar como un órgano intermedio entre la Asamblea General y el Administrador. Como cuerpo colegiado este deberá ser quien tome las determinaciones para que el administrador las ejecute. 3) El Administrador del conjunto, que ejerce operativamente la administración inmediata del mismo y tiene facultades de ejecución, conservación, representación y recaudo (art. 51). El Consejo de Administración y el Administrador deben efectuar labores interdisciplinarias encaminadas a que la personera jurídica cumpla los fines previstos en sus estatutos. Estas son las autoridades internas de las UICR 5 . (Echeverry-Velásquez y Prada-Dávila, 2017, p. 39)
\end{abstract}

Todo este contexto sobre la vida y su regulación en los multifamiliares, implica formas de relacionamiento que invitan a ser reflexionadas desde estudios sobre la vida cotidiana, donde se gestan procesos $\mathrm{y}$, en este caso, experiencias que afectan la convivencia y el ejercicio de gobierno en los multifamiliares; en ese sentido, los residentes del multifamiliar en donde se llevó a cabo la investigación, decidieron sistematizar la experiencia de un año del periodo de gobierno (2011 al 2012), en tanto los sucesos que se presentaron en este tiempo generaron conflictos y aprendizajes que, impactaron significativamente la convivencia y el ejercicio de gobierno en el multifamiliar.

En adelante se expondrá cómo se llevó a cabo la sistematización de la experiencia, mostrando para ello, en un primer apartado, los antecedentes y fundamentos de dicha modalidad investigativa; en el segundo apartado, se hará un acercamiento a los modelos de sistematización, ubicando especialmente el modelo en el cual se basó el presente ejercicio de investigación; en el tercer apartado, se muestra el desarrollo de la sistematización dando

5 Unidades inmobiliarias cerradas residenciales o multifamiliares. 
Echeverry-Velásquez y Prada-Dávila

cuenta de las diferentes fases del proceso; y en último apartado, se muestran las principales conclusiones que deja el haber realizado la sistematización, ilustrando cómo se reflejaron en el estudio realizado cada una de las dimensiones de la sistematización de experiencias.

\section{Antecedentes y fundamentos de la sistematización de experiencias como opción investigativa}

En América Latina la sistematización de experiencias se ha desarrollado desde la década del 70 del pasado siglo $X X$, en contextos varios tales como organizaciones no gubernamentales, espacios educativos informales promovidos desde la educación popular y más recientemente experiencias educativas formales, con el propósito de articular los procesos de reflexión teórica a la cualificación de las prácticas sociales. Sin embargo, "en diversos países de América Latina como Chile, Perú, México y Colombia, se empieza a escribir sobre el tema a mediados de los ochenta, en la búsqueda de conceptualizar la sistematización y hacer aportes sobre su proceso metodológico" (Cifuentes-Gil, 1999, p. 17).

Lo anterior se confirma al revisar el estado de la investigación sobre el tema en su fase heurística y encontrar literatura en este campo del conocimiento, en publicaciones de organizaciones no gubernamentales (ONG) vinculadas a experiencias de educación popular y de facultades de Trabajo Social.

Más recientemente se ha evidenciado el intercambio y socialización, mediante diversas redes, que han generado confrontaciones, debates e identificación de tendencias, que expresan el sello de las intencionalidades y los contextos en que se han desarrollado; es decir, el carácter subjetivo de la construcción teórica. (Cifuentes-Gil, 1999, p. 17)

Si bien la educación popular ha reflexionado y escrito sobre este tema "desde la relación educativa de ONG con los sectores populares, y la educación lo ha hecho desde el espacio escolar y sus búsquedas de innovación pedagógica e interacción comunitaria", es desde la academia y de manera particular desde Trabajo Social que se ha abordado la sistematización de experiencias

con una preocupación referida principalmente a la formación, a la producción teórica, a fundamentar críticamente una intervención profesional que responda a las características, condiciones, necesidades y retos del contexto, ante el agotamiento de los "modelos funcionalistas"; en tal sentido, se ha inclinado por plantear la relación entre sistematización e investigación [...] por otra parte, desde la educación popular, desde el terreno de la "práctica", se ha planteado la sistematización con la intencionalidad de recuperar las riquezas de las experiencias, la posibilidad de construir conocimiento y socializarlo. (Cifuentes-Gil, 1999, pp. 1819) 
Según Torres-Carrillo (1996), la sistematización de experiencias ha dejado de ser una temática subsidiaria de otras prácticas sociales y discursivas afines, se ha convertido en un campo del conocimiento autónomo, por cuanto convoca "sus propios discursos, instituciones, prácticas y especialistas. Según el autor en mención, "tal es el acumulado de producción acerca de la sistematización que ya empiezan a aparecer balances sobre el estado del conocimiento sobre el tema" (pp. 25-26).

No siempre que se hace referencia a la sistematización se está hablando el mismo lenguaje, por cuanto es un concepto multívoco y polifónico. "Sus acepciones han estado relacionadas con el contexto y sus desarrollos prácticos, las intencionalidades que se le otorgan y las condiciones de trabajo en que pueden realizarse" (Cifuentes-Gil, 1999, p. 27).

Según Jara-Holliday (2001), el significado otorgado comúnmente a la sistematización, hace referencia a ordenamiento, reconstrucción y tabulación de información sobre una experiencia. La connotación, menos común y más compleja, es mirar las experiencias "como procesos históricos, procesos complejos en los que intervienen diferentes actores, que se realizan en un contexto económico y social determinado y en un momento institucional del cual formamos parte". Siendo así, "el eje principal de preocupación se traslada de la reconstrucción de lo sucedido y el ordenamiento de la información, a una interpretación crítica de lo acontecido para poder extraer aprendizajes que tengan una utilidad para el futuro" (p.3).

En relación con las dimensiones de sistematización e investigación, existen diversas posiciones, algunos autores afirman que al relacionarlas se desvirtúa el sentido de la sistematización, en esta misma línea, afirma Palma (1992, p. 7) que aunque "existe diversidad de enfoques, todos postulan y fundan con claridad una identidad de la práctica sistematizadora que es distinta de la investigación". Para otros, principalmente por su oposición con los planteamientos positivistas, son prácticas opuestas; sin embargo, otros las conciben como prácticas alternativas o complementarias. Sobre esta relación complementaria Barnechea-García y Morgan-Tirado (2010) plantean lo siguiente:

\footnotetext{
La sistematización se distingue de la investigación, fundamentalmente, por su objeto: en la investigación se intenta conocer una dimensión o aspecto de la realidad, sobre la cual se formulan preguntas. En la sistematización, se hacen preguntas a una práctica, en la cual se ha participado como un actor más, con una clara intencionalidad de transformación. Como producto de la sistematización se pueden levantar preguntas de investigación que orienten a los especialistas en la producción de nuevos conocimientos directamente al servicio de la práctica y de los intereses de la transformación social. Éstos pueden constituir una referencia productiva para nuevas intervenciones y sistematizaciones, y aportar a procesos de acumulación de conocimientos adecuados para nuestra realidad. (p. 104)
} 
Finalmente, para aquellos autores, principalmente académicos, la sistematización es una investigación; por lo tanto, las consideran como procesos semejantes y equivalentes. Según Cifuentes-Gil (1999):

\begin{abstract}
La mayoría de las propuestas realizadas desde ámbitos académicos afirman la sistematización como “investigación" (Puerta Antonio, 1989), como opción investigativa (Gómez Bahena, 1991), como alternativa de investigación cualitativa, participativa y hermenéutica (Zúñiga,1995)", Hay quienes asumen la semejanza entre sistematización e investigación, pero no de corte positivista, sino, con una nueva mirada y desde otras perspectivas epistemológicas y enfoques, donde se destaca lo cualitativo, el carácter participativo, político y de transformación social. (p. 39)
\end{abstract}

Según Ghiso (2004), el interés por lo epistemológico y lo metodológico en el contexto actual, conlleva a replantear "las relaciones entre ciencia y ética y, entre conocer científico y vida". Lo anterior, hace pensar en la importancia y necesidad de propuestas alternativas de investigación social, como es el caso de la sistematización de experiencias, por medio de las cuales "se fertilice el desierto de la ciencia y haga que los hombres vuelvan a sentir el mundo como algo suyo" (p. 10). Según el autor en mención estas exigencias se presentan porque "nos estamos quedando cortos en revisiones y reivindicaciones y quizás, porque empezamos a conocer, crear y aplicar paradigmas, modelos, conceptos, discursos sin sujetos, sin historias, sin presencia y por consiguiente, sin compromisos" (Ghiso, 2004, p. $10)$.

Por lo anterior, la invitación es a generar cuestionamientos y transformaciones en los presupuestos epistemológicos y propuestas metodológicas, tomar opciones que permitan "construir, organizar y recrear conocimientos para la vida". Una propuesta epistemológica y metodológica en esta perspectiva, contiene un dialogo crítico con lo diverso y la generación de nuevas relaciones e interacciones. Para el autor en mención:

estas simientes germinan si se anidan en actitudes humanas esenciales como: indignación, autonomía, apropiación y esperanza [...] es desde estas actitudes y desarrollando procedimientos investigativos alternativos y complejos, que es posible desafiar los modos de entender lo social, retando las lógicas impuestas; reclamando, alertando y exigiendo a investigadores un discernimiento critico constante. (Ghiso, 2004, pp. 10-11) 
Echeverry-Velásquez y Prada-Dávila

\title{
3. Acercamiento a los modelos de sistematización de experiencias, al modelo y propuesta metodológica de la experiencia objeto de estudio
}

Cada experiencia es única e irrepetible, por tal razón abordar el proceso metodológico para su sistematización es un asunto complejo. Según Francke y Morgan (1995), no existen métodos universales ni únicos, es a partir de una definición clara de lo que se pretende lograr, que se debe optar entre las múltiples propuestas, por aquella que se considere pertinente.

Según Mariño y Cendales (como se citó en Cifuentes-Gil, 1999), “los modelos en lugar de ayudar empobrecen"; sin embargo, diversos autores han desarrollado propuestas metodológicas, desde diversos enfoques, con la intención de contribuir a la “operacionalización de las reflexiones y prácticas de la sistematización” (p. 73).

Según Carvajal-Burbano (2006), es necesario ver "la coherencia entre paradigma/enfoque y metodología". El optar por un enfoque empírico-analítico, criticosocial o histórico-hermenéutico, implica elegir un método en particular; "sin desconocer que también podemos transitar por paradigmas diferentes -si lo requiere el objeto de sistematización definido- pero siempre manteniendo la coherencia" (pp. 69-70).

Según Cifuentes-Gil (1999)

\begin{abstract}
Ninguna de las propuestas de sistematización ha explicitado el enfoque empírico - analítico, pues, como ya se ha afirmado, han planteado un distanciamiento de él; sin embargo, al revisar las características del lenguaje y proceso metodológico, se establece que algunas privilegian la explicación, la generalización, el conocimiento científico (pp. 55-56). (...) se ubican aquí Teresa Quiroz y María de la Luz Morgan (1988), Silva (1989), Gartner(s/f), Carlos Crespo (1989), Antonio Puerta (1989), Juan Manuel Latorre (1989), Mario Gómez Baena y el CELATS. (p. 87)
\end{abstract}

Los enfoques crítico-sociales se plasman de formas diversas en las propuestas, algunos priorizan la relación teoría-práctica; otros la relación entre construcción de conocimiento y transformación, es decir, la opción política de la sistematización; otros hablan del "método dialéctico" y las relaciones en el proceso; muchos sobre sus principios; algunos también enfatizan la reflexión crítica sobre la experiencia. En este enfoque se ubican autores como Félix Cadena, Teresa Quiroz, Antonio Ibáñez, German Mariño y Lola Cendales, entre otros.

Según Posada (como se citó en Cifuentes-Gil,1999, p. 59), la sistematización realizada desde el enfoque histórico-hermenéutico "privilegia la comprensión, la significatividad, la relevancia sociocultural del proceso de conocimiento". Para Palma (1992, p. 27) en este enfoque se ubican las propuestas que buscan obtener conocimientos a partir de la práctica, al reconstruir proyectos, identificar hipótesis, tensionar proyecto y práctica (p. 56). 
Si se opta por el enfoque hermenéutico, Carvajal-Burbano (2006) sugiere tomar como referencia las metodologías de Jara, Martinic y el PESEP. A continuación, se presentan las principales características y elementos centrales de la propuesta metodológica de sistematización del PESEP, por la que se optó en la sistematización de experiencias de la cual se da cuenta en el presente artículo.

En esta metodología la sistematización se asume como un proceso colectivo de construcción de conocimiento, que permite dar cuenta de la racionalidad interna de la experiencia estudiada y del sentido que tienen para sus actores. "Es un proceso de comprensión de sentidos en contextos específicos, en los cuales las diversas interpretaciones buscan legitimación" Zúñiga y Gómez (como se citó en Carvajal-Burbano, 2006, p. 75). Se asumen igualmente, procedimientos metodológicos de la perspectiva etnográficohermenéutica y su carácter cualitativo comprensivo.

Desde esta perspectiva, el modelo de sistematización contempla cuatro (4) dimensiones. La dimensión epistemológica busca construir conocimiento desde adentro de la experiencia, por medio de la red de interpretaciones de sus participantes, que la hacen comprensible. La dimensión ética hace referencia a la posición que los participantes asumen, ya que ellos son intérpretes de su propia realidad y en el proceso de sistematizar se reconocen entre sí como sujetos históricos. La dimensión política se refiere a la posibilidad que tienen los actores sociales de ganar espacios de poder, a las transformaciones sociales que genera el proceso de investigación, a su potencialidad para legitimar nuevos campos de saber, a la promoción del respeto a la diversidad y a legitimación del pluralismo. La dimensión pedagógica se refiere al proceso que plantea la sistematización para promover la reflexión de los actores sobre su propia experiencia, al diálogo de saberes y a la confrontación de lógicas e interpretaciones de los diversos participantes (Mulford-Ramírez, 2009).

En esta propuesta metodológica la sistematización se desarrolla mediante tres procesos:

Reconstrucción, que consiste en la reelaboración del sentido de las vivencias a partir de relatos (entrevistas, talleres, sociodramas, entre otros); interpretación, mediante la cual se construye un argumento de sentido y comprensión global de la experiencia, se establecen relaciones entre categorías significativas (lectura extensiva de relatos) y potenciación, que implica pensar la experiencia como recurso, promueve el desarrollo local y da pistas para visualizar el futuro; concreta la posición ética intrínseca en la sistematización. (Cifuentes-Gil, 1999, p. 93) 
Según Acevedo y Londoño, un aspecto a destacar en esta propuesta metodológica es el papel del macrorrelato. A este respecto afirman:

\begin{abstract}
Los relatos se asumen como unidades de análisis desde las que se intenta comprender el juego de interpretaciones que constituye la experiencia. El relato funciona como conector del recuerdo con el presente y, en cuanto construcción colectiva, logra la sanción grupal de ciertas interpretaciones, de modo que puede operar como mito legitimador de la práctica cotidiana en la experiencia[...] los relatos constituyen una historia personal de los cambios, allí los sujetos hablan sobre quiénes son y cómo han llegado a ser lo que son y sobre todo, cómo se ven y proyectan, por ello, además de los sentidos que dan a una experiencia particular, también aparecen los sentidos con los cuales colorean su vida. (como se citó en Cifuentes-Gil, 1999, pp. 95 -96)
\end{abstract}

Para los autores en mención, el proceso de sistematización implica tres tipos de lectura de los relatos:

De manera extensiva, para identificar los núcleos temáticos y la periodización endógena de la experiencia. De manera intensiva, para construir los ejes y campos semánticos o relaciones de sentido y sus desplazamientos. De manera comparativa, para identificar las perspectivas de distintos tipos de actores, lo cual da información sobre la constitución de esa perspectiva y relaciones de los actores. (Acevedo y Londoño, citado por Cifuentes-Gil, 1999, p. 96)

\title{
4. Metodología
}

\subsection{Reconstrucción, interpretación y potenciación de una experiencia en convivencia y gobierno en un multifamiliar}

De acuerdo con los desarrollos anteriores sobre la sistematización de experiencias como opción investigativa, basándose en el modelo de sistematización del PESEP, fue llevado a cabo el ejercicio de sistematización de la experiencia en convivencia y gobierno privado de un multifamiliar de la ciudad de Cali. En este apartado se expondrá el desarrollo de este ejercicio a partir de la descripción de cada una de sus fases, ilustrando las actividades, los actores involucrados, las reflexiones, y los aprendizajes que se dieron. Cabe decir que, estas fases no se dieron en una lógica precisamente secuencial, puesto que, el mismo enfoque de sistematización implica volver constantemente sobre la reconstrucción inicial de los relatos para interpretarlos en los siguientes encuentros con los actores, a la luz de sus nuevas reflexiones y generando a partir de estos escenarios de futuro, conforme se rescatan aprendizajes.

Antes de abordar el proceso de sistematización, es importante hacer alusión a las razones que incentivaron la realización de este proceso, las cuales permiten entender a su 
vez el carácter no agenciado ${ }^{6}$ de esta sistematización, pues fueron sus actores, es decir, los residentes del multifamiliar, los que reconocieron en las tensiones en la convivencia y el ejercicio de gobierno, como también en las iniciativas en pro de la convivencia y en los procesos de organización y participación comunitaria, vivencias significativas a la hora de comprender lo que sucede en el espacio en que habitan; por lo tanto, que ameritaban ser estudiadas sistemáticamente y reflexionadas críticamente. De esta forma, la sistematización surgió como una práctica no estructurada, incentivada por interrogantes que motivaron una investigación con el interés de encontrar respuestas que volvieran a la misma experiencia; en otras palabras, se buscó interpretar la experiencia considerada significativa, para movilizar reflexiones, aprendizajes y pistas que aportaran al desarrollo de buenas prácticas de convivencia y gobierno.

Lo dicho resulta representativo de las prácticas no agenciadas, al acercarse a las iniciativas y prácticas de convivencia no estructuradas que surgen en la vida cotidiana en los multifamiliares, pues por medio de estas se visibilizan modos, a través de los cuales en espacios microsociales, se construyen pedagogías movilizadoras de procesos de empoderamiento, acordes con los contextos que se habitan y de constitución de sujetos sociales y políticos conscientes de su realidad y capaces de transformarla en forma organizada.

$\mathrm{Al}$ acoger la sistematización de experiencias como una práctica investigativa que genera conocimiento, a partir de las experiencias y del sentido que sobre estas construyen los sujetos partícipes, el ejercicio de sistematización que se describe en el artículo, fue también impulsado por el interés de ahondar en el conocimiento de la relación entre la convivencia y el ejercicio de gobierno en los multifamiliares, pues al momento de realizar la investigación los acercamientos que existían sobre este campo eran en su mayoría estudios urbanísticos y arquitectónicos. En la ciudad de Cali un primer estudio que se aproximó al tema, reconociendo en los espacios multifamiliares las instancias de administración y gobierno, se encuentra en el libro Seguridad y Convivencia en Multifamiliares (Rincón-Salazar, Maldonado-Gómez y Echeverry-Velásquez, 2009), no obstante, en esta investigación previa no se establece la relación entre el ejercicio de gobierno en estos espacios cerrados residenciales y los obstáculos y facilitadores de la convivencia.

Por todo lo anterior, la sistematización de la experiencia en convivencia y gobierno privado en el multifamiliar, se desarrolló como un proceso colectivo de construcción de conocimiento, a partir de la reflexión e interpretación participativa de un hacer no estructurado, donde se reconoció el valor del proceso, se acogió la contradicción y se identificaron potencialidades.

${ }^{6}$ La sistematización agenciada se da cuando el agente que conduce y determina la misma es una institución externa a la práctica sistematizada. 
Echeverry-Velásquez y Prada-Dávila

\subsection{Reconstrucción de la experiencia en convivencia y gobierno}

Previo a la recolección de la información para la reconstrucción de la experiencia, se llevó a cabo la inserción y familiarización del equipo de investigación con la comunidad residente del multifamiliar, a través del desarrollo del proyecto "Artes en el multifamiliar", el cual tuvo como propósito el fortalecimiento de valores y habilidades artísticas de niños, niñas y jóvenes que contribuyeran a una sana convivencia y buenas prácticas de gobierno. Por medio de este proyecto se logró un acercamiento de la comunidad con el proyecto de investigación y su equipo de trabajo, venciendo las prevenciones y mitos que existen frente a la investigación social como una actividad invasiva que "usa" a la comunidad con fines meramente académicos. Este proyecto se convirtió en un espacio donde los niños, niñas y jóvenes tuvieron la oportunidad de expresarse frente a cómo los temas de convivencia y gobierno les afectan en su relación con el lugar del que también son habitantes, lo que no es habitual en los escenarios multifamiliares, donde quienes discuten estos temas son regularmente los adultos y, casi que exclusivamente, los que son propietarios. El trabajo con esta población se convirtió a su vez en un recurso para la reconstrucción de la experiencia.

En esta primera fase se construyó la unidad hermenéutica7 ${ }^{7}$ donde se agrupó toda la información representativa de la experiencia. Esta unidad estaba conformada por información documental, resultado de la revisión de 150 documentos entre circulares, comunicaciones, actas de asamblea de $\operatorname{copropietarios}^{8}$, actas de reuniones, derechos de petición y acciones de tutela, las transcripciones derivadas de la implementación de técnicas de conversación e interacción grupal como: entrevistas a profundidad a 24 residentescopropietarios (12 hombres y 12 mujeres de acuerdo con sus años de residencia y con o sin experiencia en la participación en instancias de gobierno en el multifamiliar), 6 conversatorios con residentes (primeros pobladores Torres A y B, primeros pobladores Torre C, integrantes de veeduría comunitaria ${ }^{9}$, integrantes del Consejo de administración ${ }^{10}$, equipo de investigación y académicos) así como material audiovisual de las actividades del proyecto "Artes".

7 Gestionada por medio del software de investigación Atlas Ti.

8 Ley 675 de 2001, Art 37: la asamblea general la constituirán los propietarios de bienes privados, o sus representantes o delegados, reunidos con el quórum y las condiciones previstas en esta ley y en el reglamento de propiedad horizontal. No hacen parte de esta los arrendatarios.

9 Las veedurías comunitarias son órganos de participación, exigencia, vigilancia y control al servicio de la comunidad a la que pertenece. Su labor está amparada en la Constitución Política de Colombia (Const., 1991, art. 40, 270) y en la Ley 134 de 1994, Ley 136 de 1994 y en la Ley 489 de 1998 entre otras normas.

10 Ley 675 de 2001, Art 53: los edificios o conjuntos de uso comercial o mixto, integrados por más de treinta (30) bienes privados excluyendo parqueaderos o depósitos, tendrán un consejo de administración, integrado por un número impar de tres (3) o más propietarios de las unidades privadas respectivas, o sus delegados. En aquellos que tengan un número igual o inferior a treinta (30) bienes privados, excluyendo parqueaderos y depósitos, será potestativo consagrar tal organismo en los reglamentos de propiedad horizontal. 
Se tomó como unidad de análisis cada uno de los relatos individuales y colectivos obtenidos a partir de las técnicas mencionadas. A continuación, algunos fragmentos de los relatos de los actores en lo que respecta a sus percepciones sobre la convivencia y el gobierno en la experiencia sistematizada ${ }^{11}$.

\begin{abstract}
Convivencia no es el chisme, ni que yo le voy a solucionar porque el señor tocó hasta por la noche, bien tarde, parte de conciliar con las personas, pero es promover otras cosas, cultura, educación, participación, yo sé que eso no es fácil, pero hay sitios donde lo logran. (Mujer, 1 año de residencia, sin experiencia en gobierno privado, en entrevista)

En la administración se tragan las cartas que uno les manda, aquí no aprecian la opinión de los demás, tiene que ser la opinión del consejo y del administrador. La de los niños no vale, las de las demás personas no vale. Uno va a decir algo y no sirve. (Memoria de Actividad "Conociéndonos" Proyecto Artes)
\end{abstract}

En esta fase se elaboró el macrorrelato de la experiencia ${ }^{12}$, donde a partir de los relatos se narraron los hitos o sucesos significativos de la experiencia (ver Figura 1), y se muestra, a partir de su encadenamiento, el desarrollo y comprensión global de la experiencia sistematizada, desde las diferentes voces, en una narración colectiva. Los hitos de la experiencia ilustran cómo en el multifamiliar ante las actuaciones de las instancias de gobierno y administración (administración y consejo de administración) y la forma en que desarrollaban su ejercicio, se generaron acciones e iniciativas por parte de sus residentes de forma individual y organizada (comité de convivencia y veeduría comunitaria), con la intención de incidir de forma activa en las decisiones que afectaban la vida en común en el multifamiliar, generándose así, tensiones entre los actores de la experiencia (administración, consejo de administración, residentes, comité de convivencia y veeduría comunitaria) respecto a la apertura que debía tener el ejercicio de gobierno en el multifamiliar, frente a la participación activa y constante de sus residentes; es decir, una participación más allá de la asistencia anual a la asamblea de copropietarios, incluyendo por ejemplo, acciones como la vigilancia y el control por parte de los residentes.

11 A lo largo del texto se muestran fragmentos de los testimonios de los participantes de la sistematización, estos hacen parte de la unidad hermenéutica y tienen la intención de mostrar los relatos que constituyen la base para la interpretación de la experiencia, tal como se da en la investigación de corte cualitativo y comprensivo, donde se privilegian los significados expresados por los sujetos. Además, en apartados específicos estos extractos dan cuenta de la discusión o interpretación sobre los temas que fueron objeto de mayor reflexión en el marco de la reconstrucción e interpretación de la experiencia.

12 A partir del macrorrelato se elaboró un producto audiovisual donde participaron los diferentes actores, exponiendo el sentido de los hitos de la experiencia. 
Figura 1. Hitos de la experiencia.

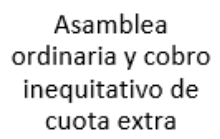

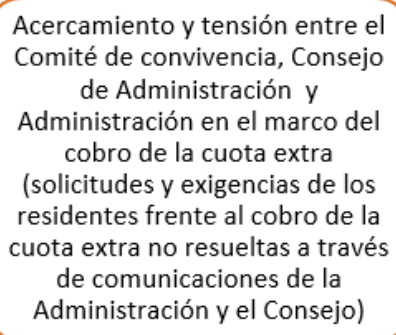

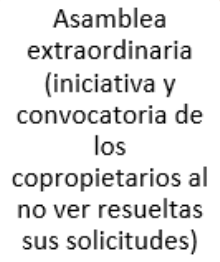

Asamblea

extraordinaria

(iniciativa y

convocatoria de los

copropietarios al

no ver resueltas

sus solicitudes)

Acciones de resistencia y protesta por parte de los residentes (abtención en el pago de cuota extra, medios de comunicación alternativos, instancias de participación y reunión alternativas)

Fuente: elaboración propia.

\section{Hallazgos}

\subsection{Interpretación de la experiencia en convivencia y gobierno}

En esta fase la experiencia narrada vuelve a los actores, por lo tanto, el producto de su narración colectiva es presentada a estos por medio de conversatorios y exposiciones de hallazgos, donde los residentes e integrantes de instancias de gobierno y administración recogieron el sentido de lo vivido, a través de las explicaciones sobre los diferentes sucesos y las reflexiones frente a estos.

Esta fase se orientó a través de las siguientes preguntas de sistematización: ¿Cuáles son las percepciones y sentidos que los residentes le otorgan a la convivencia y al ejercicio de gobierno en la experiencia?, ¿Qué tensiones y contradicciones se evidencian?, ¿Cómo los problemas de gobierno se relacionan con la convivencia?, ¿Qué prácticas sociales de resistencia son construidas por los residentes en respuesta al ejercicio del gobierno privado? y ¿Cuáles son los aprendizajes? En consecuencia, los núcleos temáticos por medio de los cuales se buscó explicitar la lógica del proceso vivido fueron: percepciones y sentidos sobre la convivencia y el ejercicio de gobierno; tensiones en la experiencia; contradicciones en la experiencia; ejercicio de gobierno privado; acciones de resistencia; y aprendizajes y pistas para la convivencia y el ejercicio de gobierno. 
En cuanto a la interpretación crítica de los mencionados núcleos temáticos, además de su elaboración interpretativa desde los sentidos construidos por los actores, se avanzó hacia su interpretación crítica por medio de la revisión de documentos académicos, otras investigaciones y bibliografía específica sobre el tema, desarrollando de esta forma reflexiones sobre los aspectos que inciden en la vida de quienes habitan y conviven en los multifamiliares, como también sobre la formas de gobierno que en estos espacios tienen lugar. En las Tablas 1, 2, 3 y 4 se exponen las principales ideas que surgen en la interpretación de los núcleos temáticos, desde los elementos teórico - conceptuales a los que se acercó el ejercicio de investigación, en la tarea de comprender una experiencia y aportar desde esta al campo de conocimiento sobre la vida en los encerramientos.

Tabla 1. Percepciones y sentidos sobre la convivencia y el ejercicio de gobierno en la experiencia.

\begin{tabular}{|c|c|}
\hline Interpretación & Interpretación crítica \\
\hline -Convivencia como & Modelo de las dimensiones de la convivencia de Giménez-Romero (2005). \\
\hline el cohabitar & Dimensiones sobre la convivencia y el ejercicio de gobierno en la experiencia \\
\hline $\begin{array}{l}\text {-Convivencia como } \\
\text { el escenario de }\end{array}$ & $\begin{array}{l}\text { Relacional: para algunos la vida en el multifamiliar sólo representa el } \\
\text { cohabitar / para otros implicó el fomento de vínculos sociales y afectivos } \\
\text { apelando a competencias ciudadanas. }\end{array}$ \\
\hline construcción de & Normativa: los problemas de convivencia y gobierno estuvieron relacionados \\
\hline fuertes vínculos, & con la baja apropiación de la norma en el contexto de vida en común. \\
\hline $\begin{array}{l}\text { donde se hacen } \\
\text { necesarias las }\end{array}$ & $\begin{array}{l}\text { Axiológica: la convivencia y el ejercicio de gobierno se afectaron cuando no } \\
\text { estuvieron basados en el reconocimiento y el respeto del otro. }\end{array}$ \\
\hline $\begin{array}{l}\text { competencias } \\
\text { ciudadanas, como }\end{array}$ & $\begin{array}{l}\text { Comunicacional: la comunicación para algunos se limitaba al saludo, y para } \\
\text { quienes incluía la discusión sobre temas del multifamiliar llegó a ser coartada }\end{array}$ \\
\hline $\begin{array}{l}\text { lo son el } \\
\text { reconocimiento y }\end{array}$ & $\begin{array}{l}\text { por instancias de gobierno, cuando no reconocían los escenarios de } \\
\text { comunicación que no eran agenciados por estas. }\end{array}$ \\
\hline el respeto del otro. & Identitaria: para algunos el único elemento vinculante fue el habitar el mismo \\
\hline -Gobierno como & $\begin{array}{l}\text { lugar, para otros el cohabitar incentivó el trabajo colectivo por la propiedad } \\
\text { común. }\end{array}$ \\
\hline un ejercicio que se & Participativa: para algunos no había tiempo para participar, y para otros que \\
\hline ve afectado por la & buscaban participar de las decisiones sobre el multifamiliar, su participación \\
\hline convivencia y los & fue coartada por instancias de gobierno, al no reconocer los escenarios que no \\
\hline vínculos que esta & eran agenciados por estas. \\
\hline propicia, en tanto & Conflictiva: los conflictos se entendieron como parte de la cotidianidad, pero \\
\hline $\begin{array}{l}\text { influye en el } \\
\text { manejo que se hace }\end{array}$ & $\begin{array}{l}\text { a su vez afectaron negativamente la convivencia y el ejercicio de gobierno por } \\
\text { el mal manejo de las tensiones. }\end{array}$ \\
\hline $\begin{array}{l}\text { de las tensiones y } \\
\text { los desacuerdos. }\end{array}$ & $\begin{array}{l}\text { Actitudinal: algunos expresaron falta de interés por lo que sucedía en su } \\
\text { entorno y otros fueron fuertemente motivados por la forma en que se } \\
\text { gestionaban las decisiones del multifamiliar, y desarrollaron iniciativas desde } \\
\text { los mismos residentes en pro de la convivencia. }\end{array}$ \\
\hline
\end{tabular}

Fuente: elaboración propia. 
Tabla 2. Tensiones y contradicciones en la experiencia-ejercicio de gobierno privado.

\section{Interpretación}

\section{Tensiones:}

- Entre los integrantes del consejo de administración a partir de posturas opuestas que, llegaron a formas de relacionamiento poco civilistas. Esto fue percibido como una situación de ingobernabilidad.

- Entre el consejo de administración y los residentes integrantes del comité de convivencia ${ }^{13}$ por cobro inequitativo de cuota extra ${ }^{14}$.

- Entre el consejo de administración y los residentes integrantes de la veeduría comunitaria por el no reconocimiento de estas instancias (comité de convivencia y veeduría) como legítimos y representativos de la comunidad y de las funciones de estas mismos en torno a las diversas situaciones del multifamiliar

- Entre residentes e instancias de administración y gobierno por iniciativas de los primeros relacionadas con: acciones de vigilancia y control; desarrollo de medios de comunicación alternativos y reclamación de derechos por vías jurídicas.

\section{Contradicciones:}

- La veeduría comunitaria fue reconocida e inscrita por la Personería Municipal de Santiago de Cali, no obstante, esta misma instancia presentó un concepto según el cual la veeduría no tenía como ámbito de actuación el contexto del multifamiliar, por ser las veedurías un mecanismo de participación ciudadana para el control tan solo a los bienes públicos, y las unidades residenciales son

\section{Interpretación crítica}

Tipos de gobierno y modelos de gobernación (Pascual, 2011).

Los tipos de gobierno o modelos de gobernación se definen según la articulación que se dé entre las tres funciones del gobierno: (legalnormativa; provisión y gestión; y relacional-participación ciudadana) y la función que adquiera mayor preponderancia, que es a su vez la función en la que se enmarcan las relaciones entre el gobierno y la ciudadanía.

El ejercicio de gobierno en la experiencia se caracterizó por:

- Tener la norma como elemento protagónico y con valor definitivo.

- Estar centrado en la función legal normativa (regulación, legislación y justificación de la gestión en los marcos).

- Hacer de la norma una camisa de fuerza que no permitía revisar errores de gestión.

- El desconocimiento de la jerarquía normativa (marcos legales como la Constitución Política y los Derechos Humanos que se encuentran por

${ }^{13}$ Ley 675 de 2001, Art 58: cuando se presente una controversia que pueda surgir con ocasión de la vida en edificios de uso residencial, su solución se podrá intentar mediante la intervención de un comité de convivencia elegido de conformidad con lo indicado en la presente ley, el cual intentará presentar fórmulas de arreglo, orientadas a dirimir las controversias y a fortalecer las relaciones de vecindad. Las consideraciones de este comité se consignarán en un acta, suscrita por las partes y por los miembros del comité y la participación en él será ad honorem.

14 Ley 675 de 2001, Art 29: los propietarios de los bienes privados de un edificio o conjunto estarán obligados a contribuir al pago de las expensas necesarias causadas por la administración y la prestación de servicios comunes esenciales para la existencia, seguridad y conservación de los bienes comunes. Estas se pagan por coeficientes, entre las funciones de la asamblea de copropietarios se encuentra: Ley 675 de 2001, Art 38: aprobar el presupuesto anual del edificio o conjunto y las cuotas para atender las expensas ordinarias o extraordinarias. Ley 675 de 2001, Art 25: todo reglamento de propiedad horizontal deberá señalar los coeficientes de copropiedad de los bienes de dominio particular que integran el conjunto o edificio. La cuota extra de la que se habla en la experiencia no fue liquidada por coeficientes, por eso era considerada inequitativa. 
Echeverry-Velásquez y Prada-Dávila

representadas como bienes privados a pesar de su propiedad común.

- Ante la presentación de acciones jurídicas (derechos de petición ${ }^{15}$, y acciones de tutela ${ }^{16}$ ) por parte de los residentes e instancias de administración y gobierno en medio de las tensiones que se presentaron, hubo decisiones y respuestas ambiguas por parte de los juzgados.

- Relación de las instancias de administración y gobierno con la norma según interés, de forma que argumentaban la imposibilidad de revisar decisiones por ser tomadas en la asamblea ordinaria de copropietarios, siendo este el máximo órgano de decisión, pero a su vez destituyeron el comité de convivencia y asignaron un revisor fiscal ${ }^{17}$ siendo esto competencia de la asamblea, tal como está reglamentado por la ley de propiedad horizontal.

\section{Ejercicio de gobierno privado en la experiencia:}

Basado en un modelo de gobierno y administración que se recogía tajantemente en la norma, se limitaba a regular y gestionar, permitiendo y promoviendo tan solo los espacios $\mathrm{y}$ acciones contemplados en los marcos normativos tal y como estos los estipulan. Es el caso de la comunicación y la participación, que se reconocían como formales en la medida en que fueran agenciadas por las instancias de administración y gobierno. Ejemplo de esto, es como el consejo de administración fundamentó en la ley de propiedad horizontal su postura de inoperatividad de la veeduría comunitaria. encima de la ley de propiedad horizontal).

- La participación se limitaba a la consulta/ los residentes eran solo contribuyentes.

- Tan solo se permitían los espacios y acciones que contempla la norma, tal y como esta los destina (comunicación formal y participación formal).

- Se descalificaba la participación de los otros cuando esta no venía de los escenarios formales o de las posturas de estos.

El tipo de gobierno (garante, racionallegal) y el modo de gobernar (Burocrático-funcionarial) que se privilegiaron en la experiencia se quedaron cortos para reconocer, entender y potenciar las dinámicas actuales de este espacio micro social en términos de participación (acciones colectivas), organización y de resistencia de sus residentes.

Fuente: elaboración propia

15 Toda persona tiene derecho a presentar peticiones respetuosas a las autoridades por motivos de interés general o particular y a obtener pronta resolución. El legislador podrá reglamentar su ejercicio ante organizaciones privadas para garantizar los derechos fundamentales (Const., 1991, art. 23).

16 Toda persona tendrá acción de tutela para reclamar ante los jueces, en todo momento y lugar, mediante un procedimiento preferente y sumario, por sí misma o por quien actúe a su nombre, la protección inmediata de sus derechos constitucionales fundamentales, cuando quiera que éstos resulten vulnerados o amenazados por la acción o la omisión de cualquier autoridad pública (Const., 1991, art. 86)

17 Ley 675 de 2001, Art 57: al Revisor Fiscal como encargado del control de las distintas operaciones de la persona jurídica. Ley 675 de 2001, Art 38: funciones de la asamblea general: elegir y remover los miembros del consejo de administración y, cuando exista, al Revisor Fiscal y su suplente, para los períodos establecidos en el reglamento de propiedad horizontal que, en su defecto, será de un año. Ley 675 de 2001, Art 58: los miembros de los comités de convivencia serán elegidos por la asamblea general de copropietarios, para un período de un (1) año y estará integrado por un número impar de tres (3) o más personas. 
Para ilustrar los diferentes sentidos sobre la experiencia en función de los roles de los actores, se exponen a continuación relatos que dan cuenta de las diferentes posturas de dos de estos, con respecto a las actuaciones en el marco del ejercicio de gobierno, específicamente en lo referente a la generación de un manual de procedimiento para la Asamblea de copropietarios elaborado por el Consejo de administración, con este se impedía el voto de los copropietarios que presentaran morosidad en sus obligaciones pecuniarias con el multifamiliar. Cabe anotar que, este manual fue elaborado posterior a la abstención en el pago de una cuota extra por parte de un grupo de copropietarios, que venían haciendo diversos reclamos a las instancias de administración y gobierno sobre este cobro, por considerarlo inequitativo (al no ser liquidado por coeficiente), al no encontrar respuesta y solución a sus reclamos, acogieron el no pago de la cuota como acto de resistencia y protesta.

En el manual de procedimiento propuesto a la próxima asamblea se recomienda limitar solamente el derecho al voto, más no al de voz y mucho menos prohibir a los morosos ingresar a la asamblea general. Con el hecho de que no puedan votar es suficiente para impedir que eventualmente puedan interferir en las decisiones de la asamblea. (Tomado de comunicación de consejo de administración del conjunto residencial contexto del estudio dirigida a comunidad en general)

(...) En esta ocasión queremos llamar la atención, con sentido de alerta e información, sobre la improcedencia e ilegalidad de la reglamentación denominada manual de procedimiento o reglamento para la asamblea general de copropietarios 2012 (...). Al examinar este documento, que viola no solo la Constitución Política, la Ley 675 de 2001, nuestro reglamento de propiedad horizontal, sino las normas más elementales de la democracia participativa en Colombia, y que cercena los derechos de libertad de expresión, participación, decisión, opinión. (Tomado de boletín No.7 veeduría comunitaria del Conjunto Residencial contexto del estudio dirigido a la comunidad). 
Tabla 3. Acciones de resistencia en la experiencia

\begin{tabular}{|c|c|}
\hline Interpretación & Interpretación crítica \\
\hline $\begin{array}{l}\text { - Organización, movilización } \\
\text { y desobediencia civil frente } \\
\text { al cobro de la cuota extra por } \\
\text { parte de los residentes. } \\
\text { - Convocatoria de asamblea } \\
\text { extraordinaria }{ }^{18} \text { por } \\
\text { iniciativa de copropietarios. } \\
\text { - Medios de comunicación } \\
\text { masivos alternos a los } \\
\text { reconocidos como formales y } \\
\text { gestionados por las } \\
\text { instancias de administración } \\
\text { y gobierno. } \\
\text { - Constitución de veeduría } \\
\text { comunitaria. }\end{array}$ & $\begin{array}{l}\text { La desobediencia civil se refiere a una } \\
\text { actuación de tipo pública y no violenta, que } \\
\text { se recoge en acciones políticas de resistencia } \\
\text { civil, estas no son opuestas a la ley y tienen la } \\
\text { intención de generar un cambio en el plan de } \\
\text { gobierno. La desobediencia civil tiene cabida } \\
\text { exclusivamente en un régimen democrático, } \\
\text { donde sin embargo ocurren violaciones a la } \\
\text { justicia y por lo tanto la desobediencia civil } \\
\text { se levanta ante la autoridad democrática } \\
\text { legítimamente elegida y reconocida (Rawls, } \\
\text { 2010). } \\
\text { En el ejercicio de gobierno de la experiencia, } \\
\text { se limitaban los mecanismos para la } \\
\text { expresión de la ciudadanía y para el ejercicio } \\
\text { del control directo, lo cual abrió la } \\
\text { posibilidad a la desobediencia civil; en la } \\
\text { medida en que cerraron las puertas ante } \\
\text { prácticas sociales asumidas por los } \\
\text { residentes para la toma de decisiones, que } \\
\text { iban más allá de las prácticas electorales; } \\
\text { tales como la organización y la movilización. } \\
\text { Tal desobediencia civil se expresó por medio } \\
\text { de las acciones de resistencia, las cuales } \\
\text { tuvieron como fundamentos los Derechos } \\
\text { Humanos, la Constitución Política, la } \\
\text { democracia y el bien común. }\end{array}$ \\
\hline
\end{tabular}

Fuente: elaboración propia

18 Ley 675 de 2001, Art 39: la asamblea general se reunirá en forma extraordinaria cuando las necesidades imprevistas o urgentes del edificio o conjunto así lo ameriten, por convocatoria del administrador, del consejo de administración, del Revisor Fiscal o de un número plural de propietarios de bienes privados que representen por lo menos, la quinta parte de los coeficientes de copropiedad. 
Echeverry-Velásquez y Prada-Dávila

Tabla 4. Aprendizajes y pistas para la convivencia y el ejercicio de gobierno

\begin{tabular}{|c|c|}
\hline Interpretación & Interpretación crítica \\
\hline $\begin{array}{l}\text { - Capacidad de } \\
\text { agencia. }\end{array}$ & $\begin{array}{l}\text { Desde el enfoque de capacidades de Martha Nussbaum } \\
\text { (2002), una de las capacidades centrales que deben tener los } \\
\text { sujetos en pos de su desarrollo humano es la de control } \\
\text { sobre el propio entorno, que en la medida en que se hace } \\
\text { efectiva, se traduce en la posibilidad de participación } \\
\text { política, de expresión y organización, para lo cual es clave } \\
\text { un gobierno que promueva un entorno y unas acciones de } \\
\text { cara al funcionamiento de esta capacidad. }\end{array}$ \\
\hline $\begin{array}{l}\text { - Reconocimiento } \\
\text { del marco legal de } \\
\text { la propiedad } \\
\text { horizontal. }\end{array}$ & $\begin{array}{l}\text { La capacidad de agencia fue reconocida por los actores de } \\
\text { la experiencia como un aprendizaje significativo, en tanto } \\
\text { se tradujo en la capacidad de incidir en las decisiones y } \\
\text { acciones que afectaban su entorno cotidiano, y de } \\
\text { transformarlas para acercarse a las prácticas de buen vivir } \\
\text { y ejercicios de gobierno acorde a estas. Lo anterior se } \\
\text { materializó precisamente al momento en que se hizo } \\
\text { efectivo su derecho a la participación (individual y } \\
\text { colectica), por lo cual, la defensa de derechos relacionados } \\
\text { con la oportunidad de ejercer la participación en los } \\
\text { asuntos del multifamiliar, incluso desde la organización } \\
\text { social, fue también uno de los aprendizajes. }\end{array}$ \\
\hline & $\begin{array}{l}\text { Finalmente, la revisión de los marcos normativos que } \\
\text { orientan la vida en los multifamiliares, como lo es la Ley } \\
675 \text { de } 2001 \text { de propiedad horizontal y el reglamento de } \\
\text { propiedad horizontal del multifamiliar contexto de la } \\
\text { investigación, a la luz de lo vivido y desde de la } \\
\text { sistematización de la experiencia, permitió comprender a } \\
\text { los actores que estos marcos se han quedado cortos para } \\
\text { entender las nuevas dinámicas que se presentan en estos } \\
\text { contextos y que sobre todo no logran potenciar las prácticas } \\
\text { ciudadanas que nacen en estos, como son la organización } \\
\text { de la comunidad y la participación colectiva. }\end{array}$ \\
\hline
\end{tabular}

Fuente: elaboración propia 


\subsection{Potenciación de la experiencia en convivencia y gobierno}

En el proceso de sistematización afloraron aprendizajes y pistas que los actores encontraron como aspectos necesarios para avanzar hacia buenas prácticas de convivencia y gobierno en el multifamiliar, incluso destacaron el impacto de la experiencia en esas actuales prácticas, pues algunas lograron transformarse en medio del mismo proceso de sistematización; por ejemplo, en lo que respecta a las formas de relacionarse entre actores con posturas opuestas, quienes llegaron a trabajar en conjunto en las instancias de administración y gobierno o demás instancias (comité de convivencia y veeduría comunitaria).

La potenciación en la sistematización de experiencias se dio en tres sentidos: el reconocimiento de las potencialidades que representa en sí el vivir en encerramientos residenciales; lo que potenció la experiencia en términos de aprendizajes y pistas para la convivencia y el ejercicio de gobierno; y lo que potenció en el contexto de la investigación el haber realizado el ejercicio de sistematización.

Potencialidades que representan en sí la vida y el ejercicio de gobierno en un multifamiliar

Si bien los problemas de convivencia y gobierno fueron parte de lo que motivó la sistematización de experiencias, el haber reflexionado sobre lo vivido pensando en lo que significa el modo de vida en los encerramientos residenciales, les permitió a los actores identificar en el contexto de los multifamiliares aspectos que favorecen el desarrollo de buenas prácticas de convivencia y gobierno.

- Los multifamiliares son escenarios que demandan y promueven la participación para la toma de decisiones sobre una propiedad común, lo cual conlleva prácticas que sirven de base a la socialización de competencias ciudadanas.

- Por ser los multifamiliares espacios de residencia, existen arraigos hacia los bienes tangibles e intangibles que estos representan, lo cual históricamente ha movilizado la participación y organización ciudadana.

- En los multifamiliares llegan y residen personas con deseo de trabajar por los mismos y por ende con la motivación de hacer parte de las instancias de gobierno y demás colectivos. 
Potenciación de la experiencia sistematizada

Lo vivido resultó en los siguientes aprendizajes y pistas para avanzar hacia el desarrollo de buenas prácticas de convivencia y gobierno.

- Se reconoció la necesidad de abrir instancias diferentes a las reconocidas en el ejercicio formal de gobierno, para hacer frente a las demandas de participación y organización abierta, directa y colectiva.

- Se trascendió hacia la acción colectiva como una de las vías legítimas de participación.

- Se reconoció la necesidad de revisar no solo la gestión de los dineros del multifamiliar, sino los estilos de gobierno y las formas en que se interviene la convivencia.

- Surgieron estilos de gobierno que hablan de intereses comunitarios, como de enfrentar el conflicto sin la trasgresión de ese otro con una postura contraria.

- Se manifestó la importancia de construir administraciones y gobiernos que trabajen también la dimensión de lo social, y no exclusivamente la provisión de bienes y servicios, así como el control de los recursos.

- Se reconoció la relación de afectación entre los estilos de gobernar y la convivencia.

- Se estableció la importancia de revisar los marcos normativos que rigen la convivencia y el gobierno en los multifamiliares, a la luz de situaciones que sobrepasan estos marcos, llegando incluso a pensar en la importancia de hacer revisiones a nivel de la ciudad que puedan derivar en lineamientos de política pública, teniendo en cuenta que el encerramiento residencial es hoy el mayormente ofertado por el sector de la construcción.

\section{Potenciación a partir del ejercicio de sistematización}

El significado de las situaciones que se dieron durante el periodo estudiado, motivó la participación de los actores en el ejercicio de sistematización, pues al haber cuestionado y reconocido las implicaciones de las prácticas de gobierno y su relación con la convivencia, encontraron pertinente hacer un proceso de reflexión de tales situaciones, que a su vez les 
Echeverry-Velásquez y Prada-Dávila

aportara nuevos aprendizajes para aplicar en la gestión de la convivencia y el gobierno en el multifamiliar.

De igual forma, la reciprocidad frente al ejercicio de investigación fue favorecida por la familiarización del estudio y del equipo de investigación con el contexto del multifamiliar, a través del proyecto "Artes", donde se logró mostrar a los habitantes de este escenario cómo desde la sistematización de experiencias se promueven estrategias de investigación que no se quedan en "usar" a las comunidades con fines exclusivamente académicos, sino que trascienden hacia el conocimiento para la vida, un saber que vuelve a la práctica.

La sistematización además, fue en sí misma facilitadora tanto de la participación de los actores, como de la posibilidad de reflexionar sobre la experiencia y extraer aprendizajes de esta para la práctica pues, partiendo de su apuesta epistemológica, se desarrolló una estrategia metodológica que propició el encuentro, diálogo y reflexión entre los habitantes del multifamiliar, abonando así el camino hacia nuevas formas de relacionamiento (diálogo crítico y diálogo con lo diverso), promoviendo la comprensión de aspectos que si bien son habituales, afectan significativamente la vida en comunidad. A continuación, algunos de los aspectos que potenció la sistematización en la experiencia.

- $\quad$ Se reconoció el modo de gobernar como un ejercicio que hace parte de la vida cotidiana.

- Se consideraron los multifamiliares como un escenario de intervención social.

- Se abrió la puerta a nuevas estrategias de relacionamiento con los actores y se dio voz a los que no la tenían, como los niños, niñas y jóvenes que participaron en el proyecto "Artes".

- Se reconoció la presencia de procesos comunitarios en escenarios que trascienden la mirada tradicional de este concepto.

- Se potenció la reflexión de los actores frente a lo que implica vivir en un multifamiliar.

- Permitió que las personas de la comunidad se dieran cuenta que la convivencia va más allá de los eventos sociales.

- $\quad$ Se verbalizó el significado del proceso vivido. 


\section{Conclusiones}

Es preciso hacer algunos apuntes finales sobre el ejercicio de investigación expuesto, reconociendo en este un proceso que da lugar a la reflexión sobre la sistematización de experiencias como opción investigativa, que se nutre del método cualitativo para aportar al conocimiento de los fenómenos de la vida cotidiana desde las representaciones que sobre estos construyen los sujetos que los experimentan, a partir de su interpretación crítica, donde tienen cabida las relaciones con el contexto histórico, las condiciones sociopolíticas y los otros marcos y estudios que han buscado dar explicaciones a dichos fenómenos.

En esta perspectiva el sujeto que experimenta el fenómeno social estudiado, para el caso, los procesos ligados al ejercicio de gobierno privado en un multifamiliar, recibe la connotación de actor, lo cual representa su lugar activo tanto en la construcción como en la reflexión sobre la experiencia; este es un actor que comparte sus saberes sobre la experiencia y que se permite construir aprendizajes en la medida en que interactúa con los saberes de los otros actores. Cabe añadir que, los investigadores desde la postura epistemológica de la investigación cualitativa, son también actores de la experiencia, en tanto llegan a ser parte del proceso de reflexión y aportan a este desde sus experiencias ligadas a otros contextos y culturas, y desde sus propios saberes y prácticas investigativa y académica.

Haciendo referencia a la fuerza de potenciación de la sistematización de experiencias, esta logró detonar las dimensiones epistemológica, pedagógica, política y ética. En términos de la dimensión epistemológica la sistematización se acercó a la práctica para construir un conocimiento sobre esta, propiciando su comprensión desde y para sus mismos actores. En concordancia, desde la dimensión ética, los conocimientos generados desde la experiencia volvieron a los actores para convertirse en nuevas prácticas, claro está, ligadas a sus posturas sobre lo que reconocen, con base en lo vivido y reflexionado, como buenas prácticas de convivencia y gobierno, entre estas las que apuestan por la inclusión, el reconocimiento del otro, la defensa del interés general y la construcción de lo común.

En lo concerniente a la dimensión pedagógica, todo el aprendizaje y aporte al conocimiento se dio de la mano de un ejercicio de sistematización que propició el diálogo de saberes y el encuentro y desencuentro de interpretaciones diversas, al reconocerse los diferentes roles que tuvieron lugar en la experiencia y su relación con las posturas asumidas. Se promovió la reflexión sobre la experiencia de actores diversos con estrategias de conversación colectiva, encuentros lúdicos-pedagógicos y revisión de hallazgos. Estas mismas actividades aportaron aprendizajes sobre el manejo del conflicto entre sujetos con posturas contrarias, además, de brindar herramientas para reconocer la pertinencia de formas de participación pluralistas. La dimensión política se detonó a través de transformaciones en las prácticas de convivencia y gobierno en el multifamiliar, se generaron nuevos espacios de participación e incidencia de los residentes en el gobierno, 
estos espacios se caracterizan por: la trascendencia de la acción individual a la colectiva; las acciones ciudadanas de vigilancia, control y exigencia; la legitimidad de las acciones de resistencia; el respeto a la diversidad; y el reconocimiento del pluralismo. Todo esto resultado de la reflexión sobre los marcos normativos que fundamentan el gobierno privado y su relación con la democracia, desencadenando en el empoderamiento de los sujetos y el fortalecimiento de su capacidad de agencia.

Si bien la investigación que dio origen al presente artículo no se llevó a cabo en tiempo de pandemia, es innegable que su publicación se realizará en esta coyuntura presente en todos los ámbitos de la geopolítica mundial. Por lo anterior, es importante reconocer la significación y pertinencia de continuar ahondando en el estudio de los problemas de la convivencia, la gobernabilidad y otros asuntos asociados al habitar contemporáneo en estos espacios de vivienda multifamiliar, por cuanto, en esta coyuntura de pandemia el confinamiento obligatorio o voluntario de la población, ha conllevado a la permanencia y desarrollo de la vida social y laboral en el lugar de residencia y al fortalecimiento de los sistemas de regulación (legal, social, moral/autocontrol), por parte de las instancias de administración y gobierno privado de estos espacios habitacionales y de sus residentes. La conjugación de estos aspectos señalados ha desencadenado nuevas formas de relacionamiento social y problemas de convivencia, que ameritan ser estudiados para su potenciación en el caso de las buenas prácticas de convivencia o adecuada gestión en el caso de los conflictos. En este marco de ideas, la sistematización de experiencias como opción investigativa, desde el modelo propuesto en la experiencia estudiada, es una opción y alternativa válida.

\section{Referencias bibliográficas}

Barnechea-García, M. M., y Morgan-Tirado, M. L. (2010). La sistematización de experiencias: producción de conocimientos desde y para la práctica. Tendencias y Retos, (15), 97-107. Carvajal-Burbano, A. (2006). Teoría y práctica de la sistematización de experiencias. Cali, Colombia: Universidad del Valle. Facultad de Humanidades. Escuela de Trabajo Social y Desarrollo Humano.

Cifuentes-Gil, R. M. (1999). La sistematización de la práctica en Trabajo Social. Buenos Aires, Argentina: Lvmen.

Congreso de Colombia. (2 de junio de 1994). [Ley 136 de 1994]. DO: 41.377.

Congreso de Colombia. (29 de diciembre de 1994). [Ley 489 de 1994]. DO: 43.464.

Congreso de Colombia. (3 de agosto de 2001). Ley de Propiedad Horizontal. [Ley 675 de 2001]. DO: 44.509.

Congreso de Colombia. (31 de mayo de 1994). [Ley 134 de 1994]. DO: 41.373.

Constitución Política de Colombia [Const]. (1991). 2da Ed. Legis 
Echeverry-Velásquez y Prada-Dávila

Echeverry-Velásquez, M. L. y Prada-Dávila, M. (2017). Gobernabilidad y desobediencia civil en unidades inmobiliarias cerradas residenciales (UICR). Una experiencia de gobierno privado en un multifamiliar de la ciudad de Cali. Revista Eleuthera, 16, 32-53.

Francke, M., y Morgan, M. L. (1995). La Sistematización: apuesta por la generación de conocimientos a partir de las experiencias de promoción. Recuperado de http:/ / centroderecursos.alboan.org/ebooks/0000/0738/6_ESC_SIS.pdf.

Ghiso, A. (2004). Entre el hacer lo que se sabe y el saber lo que se hace. Una revisión sui géneris de las bases epistemológicas y de las estrategias metodológicas. Aportes, (57), 9-22.

Giménez-Romero, C. (2005). Convivencia. Conceptualización y sugerencias para la praxis. Puntos de Vista. Cuadernos del Observatorio de las Migraciones y de la Convivencia Intercultural de la Ciudad de Madrid, (1), 7-32.

Jara-Holliday, O. (abril, 2001). Dilemas y desafios de la sistematización de experiencia. En Seminario ASOCAM: Agricultura sostenible campesina de montaña. Intercooperation, Cochabamba, Bolivia.

Mulford-Ramírez, E. J. (2009). Somos gente de procesos: un estudio sobre el reconocimiento de derechos individuales y colectivos a partir de la sistematización de una experiencia de formación de promotores jurídicos en derecho social. Cali, Colombia: Corporación Asesorías para el Desarrollo. ASDES.

Nussbaum, M. (2002). Las Mujeres y el desarrollo Humano. Barcelona, España: Herder.

Palma, D. (1992). La Sistematización como estrategia de conocimiento en la educación popular. El estado de la cuestión en América Latina. Santiago, Chile: CEAAL.

Pascual, J. M. (2011). El papel de la ciudadanía en el auge y decadencia de las ciudades. El fin del gerencialismo o la recuperación de lo público y sus actores. Valencia, España: Tirant lo Blanch.

Rawls, J. (2010). Teoría de la Justicia. México: Fondo de Cultura Económica.

Rincón-Salazar, M. T., Maldonado-Gómez, M. C., y Echeverry-Velásquez, M. L. (2009). Seguridad y convivencia en multifamiliares. Una mirada al encerramiento residencial. Cali, Colombia: Universidad del Valle. Facultad de Humanidades. Escuela de Trabajo Social y Desarrollo Humano.

Torres-Carrillo, A. (1996). La Sistematización desde la perspectiva interpretativa. Aportes, (44), 23-37. 


\section{OTROS ARTÍCULOS DE PROSPECTIVA No. 31 DE 2021}

\section{PRESENTACIÓN}

Presentación. Reflexiones sobre desafios al publicar sistematizaciones

Rosa María Cifuentes-Gil

\section{EDITORIAL}

Reflexiones sobre Trabajo Social: aportes de la Sistematización

María Rocío Cifuentes-Patiño

\section{ARTÍCULOS}

Hacer lo que se sabe, pensar lo que se hace. La sistematización como modalidad investigativa Alfonso Torres-Carrillo

Aportes y desafios de la Sistematización de experiencias en el Trabajo Social y la extensión crítica. Apuntes y reflexiones desde la perspectiva de la Educación Popular

María Rosa Goldar

Valeria Chiavetta

La sistematización en Trabajo Social y la epistemología feminista del punto de vista. Diálogos sobre la producción de conocimiento sustentada en experiencias

Ruth Noemí Parola

María Florencia Linardelli

La Sistematización investigativa de las experiencias: del baile de los que sobran a la fiesta de los que faltan

María Belén Ortega-Senet

Sistematización y Trabajo Social en Chile. El largo y sinuoso camino

Patricia Lorena Castañeda-Meneses

Ana María Salamé-Coulon
Sentipensar la pandemia COVID-19 desde la sistematización de la experiencia en Trabajo Social: reflexiones del profesor Oscar Jara Holliday

Elia Sepúlveda-Hernández

La sistematización de experiencias, una investigación social cualitativa que potencia buenas prácticas de convivencia y gobierno. La experiencia de un conjunto residencial multifamiliar en Cali, Colombia Martha Lucia Echeverry-Velásquez Manuela Prada-Dávila

Construcción de subjetividades epistemológicaspolíticas de profesoras y profesores de Investigación social en una universidad privada y confesional en Bogotá

Giovanni Mora-Lemus

Sistematización de la experiencia Reconocimiento de los derechos humanos del adulto mayor en dos familias residentes en Cali y Valledupar (Colombia)

Lina María Cuello-Lacouture

Jimena del Pilar Jaramillo-Jaramillo

La memoria transformadora como estrategia de intervención profesional en los procesos de reconciliación social: comprensión a partir de mujeres campesinas, excombatientes y jóvenes en Manizales, Colombia

Yeimmy Stephania Corredor-Sotelo

Juliana Fuertes-Fuertes

Sistematización de una estrategia de educación informal implementada en personas privadas de la libertad en el establecimiento penitenciario de mediana seguridad y carcelario de Barranquilla, Colombia

Rafael Humberto Herrera-Mercado Rafael Alberto Zambrano-Vanegas 
Aportes significativos del proceso de intervención comunitaria con la Escuela Popular de Comunicación Alternativa Jaime Garzón de la ciudad de Cúcuta, Colombia

Carlos Lasso-Urbano

La sistematización de la intervención como metodología de investigación en Trabajo Social. Importancia práctica y teórica de la fase de recogida de datos en la intervención social según experiencia del Programa de Apoyo a las Familias en Zaragoza, España

Elisa Esteban-Carbonell

Nuria Del Olmo-Vicén

Papel de la sistematización de experiencias en los procesos de evaluación de intervenciones de salud pública en la Comuna Saludable por la Paz, Cali - Colombia

Jenny Faisury Peña-Varón

Paola Andrea Marín-Velásquez

Janeth Mosquera-Becerra

Experiencia de intervención social en hogares comunitarios integrales del barrio Alfonso Bonilla Aragón, Cali - Colombia

Julián Alexander Montaño-Cárdenas

Las políticas sociales y el gobierno de la "población indígena". Estrategias y regulaciones en el multiculturalismo chileno

Rodrigo Agustín Navarrete-Saavedra

Representaciones sociales sobre estilo de autoridad y tipos de interacción en cuidadores de residencias de protección infantil en Chile

Marcelo Gallegos-Fuentes
Carmen Gloria Jarpa-Arriagada

Reflexiones sobre inseguridad social y cuestiones penales. Una respuesta estratégica a partir de experiencias de cooperativismo con ex detenidos en Argentina

Analia Elizabeth Otero

Yael Yanina Barrera

Desarrollo y salud: la emergencia de un nuevo paradigma

Jesús María Sánchez-Ordóñez

Trabajo Social en ejercicio libre: la perspectiva profesional en España

Paula Frieiro-Padín

Tamara Fernández-Arias

Rubén González-Rodríguez

\section{RESEÑAS}

Social Work and the City: Urban Themes in 21stCentury Social Work

Felipe Saravia-Cortés

Respuestas del Trabajo Social ante emergencias sociales y problemáticas sociales complejas de México y España

Felipe Saravia-Cortés

El feminismo, el género y la profesionalización del trabajo social en Colombia (1936-2004)

Ambar Oriana Serna-Lombo

El puño invisible. Arte, revolución y un siglo de cambios culturales

Carlos Arturo Robledo-Marín

\section{PROSPECTIVA}

No. $31 \bullet$ ene.-jun. 2021

e-ISSN: 2389-993X • Universidad del Valle 